\title{
Temporal solar irradiance variability analysis using neural networks
}

\author{
Ambelu Tebabal ${ }^{1}$, Baylie Damtie ${ }^{1}$ and Melessew Nigussie ${ }^{1}$ \\ ${ }^{1}$ Washera Geospace and Radar Science Laboratory (WaGRL),Bahir Dar University, Bahir Dar \\ Ethiopia \\ email: ambelut@bdu.edu.et
}

\begin{abstract}
A feed-forward neural network which can account for nonlinear relationship was used to model total solar irradiance (TSI). A single layer feed-forward neural network with Levenbergmarquardt back-propagation algorithm have been implemented for modeling daily total solar irradiance from daily photometric sunspot index, and core-to-wing ratio of $\mathrm{Mg}$ II index data. In order to obtain the optimum neural network for TSI modeling, the root mean square error (RMSE) and mean absolute error (MAE) have been taken into account. The modeled and measured TSI have the correlation coefficient of about $\mathrm{R}=0.97$. The neural networks (NNs) model output indicates that reconstructed TSI from solar proxies (photometric sunspot index and $\mathrm{Mg}$ II) can explain $94 \%$ of the variance of TSI. This modeled TSI using NNs further strengthens the view that surface magnetism indeed plays a dominant role in modulating solar irradiance.
\end{abstract}

Keywords. Sunspots, Neural networks

\section{Introduction}

Total solar irradiance (TSI) is the total power from the sun impinging on the Earth and its variation in solar radiative output clearly has the potential to affect our planet. This radiative output heats the land and ocean, maintains our atmosphere, generates clouds, and cycles water (Rottman, 2006). These influence mechanisms of the sun can be linked to solar variations of luminosity, magnetic field, UV radiation, solar flares and modulations of cosmic ray intensity (Pap \& Fox, 2004; Lean, 2001).

Following the satellite era, various empirical and semi-empirical models have been developed to model total solar irradiance from solar activity. Authors like Fröhlich \& Lean (2004); Balmaceda et al. (2007) used empirical TSI modeling to address the question of the extent to which variations in TSI can be explained using only variations due to magnetic features such as sunspots, faculae and network. The fact that the TSI is on average higher near sunspot maximum implies that the influence of faculae and network is greater than that of sunspots (Fröhlich \& Lean, 1998). Proxy-based models (see, Ashamari et al., 2015, for a recent review) try to answer the question: to what extent specific solar proxies, such as sunspot number, Mg II index, F10.7 radio flux, abundance of cosmogenic isotopes, spectral lines or specific wavelength bands in the observed solar spectra or solar intensitygrams, can be used to describe solar irradiance variability.

Neural Networks (NNs) have been widely used in multivariate nonlinear time series modeling in many research areas such as electronics, aerospace, and manufacturing engineering. $\mathrm{NN}$ is capable of directly correlating the time series of multiple input variables to the output variables through the interconnected nodes using trainable weights and bias signals (Hagan et al., 1995). 
In this study, our objective was to develop a neural network model to forecast SOURCE TSI from measurements of solar proxy data. We consider the separate correspondence between the short- and long-term components of Mg II and solar irradiance time series. Fröhlich \& Lean (2004) pointed out that the contribution of bright regions (i.e. faculae and network) to the TSI can be better modeled by a three component model where the short- and long-term components of Mg II have separate coefficients rather than by a single Mg II term.

\section{Data}

The input data have been taken from the solar indices that have impact on the TSI variations such as the daily PSI, and Mg II index. The daily PSI indicates the solar activity and it has been compiled by Balmaceda, Solanki \& Krivova (2009) and downloadable at: http://www2.mps.mpg.de/projects/sunclimate/data/table4_v0613.txt. The Solar Radiation and Climate Experiment (SORCE) Mg II Index is available at : http://lasp.colorado.edu/lisird/tss/composite_mg_index_csv?. This index is defined as the ratio of the irradiance in the core of the unresolved $\mathrm{Mg}$ II doublet at $280 \mathrm{~nm}$ to the nearby continuum irradiance and measures solar variability on both rotational and solar cycle time scales ( Deland \& Cebula, 1993). All these indices are considered as an input parameter for TSI modeling by considering their short term as well as long term influences on solar activity. The Total Irradiance Monitor (TIM), a radiometer on the SORCE satellite, has been observing TSI since February 2003 with an absolute accuracy of $350 \mathrm{ppm}$, an instrumental noise of $12 \mathrm{ppm}$ and a stability of better than $10 \mathrm{ppm} / \mathrm{yr}$ (Kopp \& Lean, 2011). In this study we use version 17, level 3 six-hourly data averaged onto a daily cadence. The database we use extends over the period 2003 to 2013 with a total data of 3461. Figure 1 shows daily values from top to bottom: the SORCE of the TSI, PSI, Mg $\mathrm{II}_{l t}$, and $\mathrm{Mg} \mathrm{II}_{s t}$ from the year 2003-2013 respectively

\section{Methodology}

Neural networks (NNs) are mathematical system which can be able to model the ability of biological neural networks by interconnecting many simple neurons. The neuron accepts inputs from a single or multiple sources and produces an output by training process with a predetermined non-linear function. Hornik et al. (1989) have proven that the perception with just one hidden layer is capable of approximating any measurable function to any desired degree of accuracy. For this study, mathematical representation of the perception with one hidden layer can be represented as follows:

$$
T S I_{\text {pre }}=\sum_{j=1}^{6} \hat{w}_{n j} f\left(\sum_{i=1}^{3} w_{j i} x_{i}+b_{j}\right)+\hat{b}_{n j} .
$$

where TSI Tre $_{\text {is }}$ the predicted TSI of the output neuron, $x_{i}$ is the input value and $\mathrm{i}$ goes from 1 to $3, w_{j i}$ the weights connecting the input layer to the hidden neurons and $\mathrm{j}$ goes from 1 to $6, b_{j}$ represents the bias associated to the hidden neuron, $f$ is the transfer function of the hidden neurons (here, the function chosen to be hyperbolic tangent function for all neurons), $\hat{w}_{n j}$ is the weights connecting hidden neuron to the output neuron, which contains a unique node $\mathrm{n}$, and $\hat{b}_{j}$ represents the bias associated to the output neuron. The root mean square error (RMSE) and mean absolute error (MAE) were used for the 


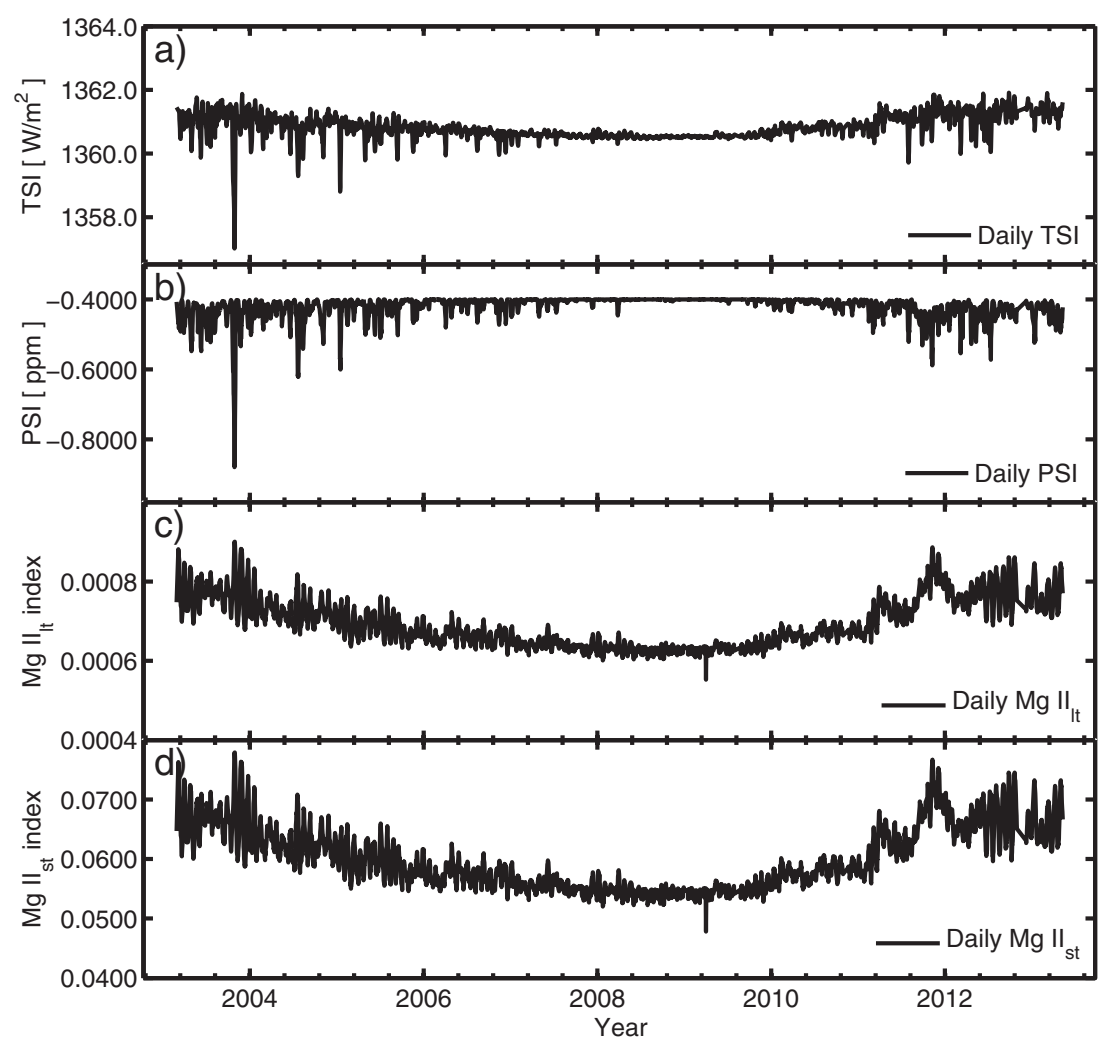

Figure 1. Daily values of the time series a) SORCE TSI b) Photometric Sunspot Index (PSI) c) $\mathrm{Mg} \mathrm{II} l t$ and d) Mg II $s t$ index. The period covers from Febuary 27, 2003 up to May 10, 2013.

agreement index to present the accuracy of the current model given as:

$$
\begin{aligned}
R M S E & =\sqrt{\frac{1}{N} \sum_{i=1}^{N}\left(T S I_{\text {meas }}^{i}-T S I_{\text {pre }}^{i}\right)^{2} .} \\
M A E & =\frac{1}{N} \sum_{i=1}^{N}\left|T S I_{\text {meas }}^{i}-T S I_{\text {pre }}^{i}\right| .
\end{aligned}
$$

where $\mathrm{N}$ is the number of data points, $T S I_{\text {meas }}$ is the measured data by using satellites, and $T S I_{\text {pre }}$ is predicted by the model.

The two primary components of the NNs are the processing elements and their interconnections. Figure 2 shows a fully connected single hidden layer feed-forward NNs and it has categories of layers in a neural network: input, hidden and output layers. The input layer receives the initial values of the variables; the output layer shows the results of the network for the input values; and the hidden layer performs the operations designed to obtain an output. The input layer must have as many neurons as there are input variables, and the output layer must have as many neurons as the outputs produced by the network. 
Table 1. Results of NNs techniques for training, validation and testing data set.

\begin{tabular}{ccccccc}
\hline \multirow{2}{*}{$\begin{array}{c}\text { No of } \\
\text { hidden } \\
\text { neuron }\end{array}$} & \begin{tabular}{c} 
RMSE \\
\cline { 3 - 6 }$\left(\mathrm{Wm}^{-2}\right)$
\end{tabular} & $\begin{array}{c}\text { MAE } \\
\left(\mathrm{Wm}^{-2}\right)\end{array}$ & $\begin{array}{c}\text { RMSE } \\
\left(\mathrm{Wm}^{-2}\right)\end{array}$ & $\begin{array}{c}\text { MAE } \\
\left(\mathrm{Wm}^{-2}\right)\end{array}$ & $\begin{array}{c}\text { RMSE } \\
\left(\mathrm{Wm}^{-2}\right)\end{array}$ & $\begin{array}{c}\text { MAE } \\
\left(\mathrm{Wm}^{-2}\right)\end{array}$ \\
\hline 3 & 0.1028 & 0.0736 & 0.1032 & 0.0735 & 0.1095 & 0.755 \\
4 & 0.1012 & 0.0724 & 0.111 & 0.0732 & 0.1085 & 0.0745 \\
5 & 0.1001 & 0.0726 & 0.0988 & 0.0706 & 0.1105 & 0.0728 \\
6 & 0.1007 & 0.0709 & 0.0881 & 0.0635 & 0.0919 & 0.0671 \\
7 & 0.0992 & 0.0698 & 0.0948 & 0.0573 & 0.1028 & 0.0755 \\
8 & 0.0963 & 0.0689 & 0.0986 & 0.0702 & 0.1141 & 0.0735 \\
9 & 0.0900 & 0.0698 & 0.0954 & 0.0671 & 0.1050 & 0.0709 \\
\hline
\end{tabular}

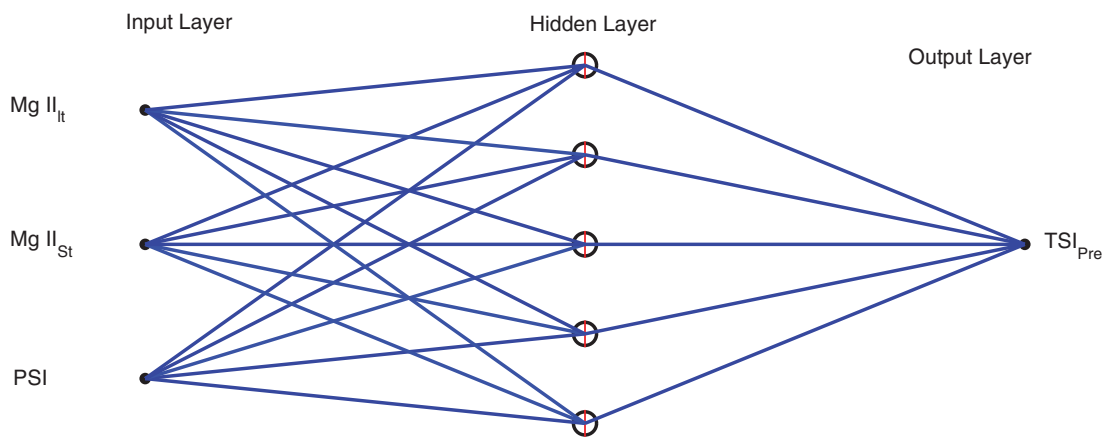

Figure 2. Schematic of a single hidden layer, feed-forward neural network to reconstruct TSI.

\section{Results}

It is known that neural networks interpolate well within the input space, and therefore the network is expected to reproduce the data set that was used to train it with relatively good accuracy (Habarulema et al., 2007 ; Habarulema \& McKinnell 2012 ; Tebabal et al., 2015). However, this model may not generalize well to new data that is outside the training set (Srivastava et al., 2014; Demuth \& Beale, 2000)). The risk of over-fitting during the training can be prevented by using early stopping techniques, accordingly the data can be randomly partitioned into training, validation and testing sets. The model building data set is used to estimate model parameters (weights and biases) and validation set is to check the performance of the network to determine the epoch at which training should be stopped to avoid over-fitting or over-training. The testing set is used to assess final overall performance of the NNs.

Comparison of model prediction and observations during training, validation and testing phases are given in Figure 3. The correlation coefficients between the model prediction and observations for both phases were $0.9648,0.964$ and 0.97 respectively. A summary of the statistics of the model performance is given in Table 1. As shown in this table the network with single hidden layer of 6 hidden neurons has achieved the best performance. Results show that the neural network model was satisfactorily trained to determine the parameters in the network so that the modeled TSI match with the SORCE TSI for testing data sets.

The temporal comparison of model prediction and SORCE TSI during testing phase is given in Figure 4. As it is seen from the figure the temporal variation of SORCE TSI is well predicted by the model. To examine the linear association between the TSI for the testing data and NNs model output, we present a scatter plot in Figure 3. The red colored dashed line represents the best fit linear regression line between the NNs prediction and 

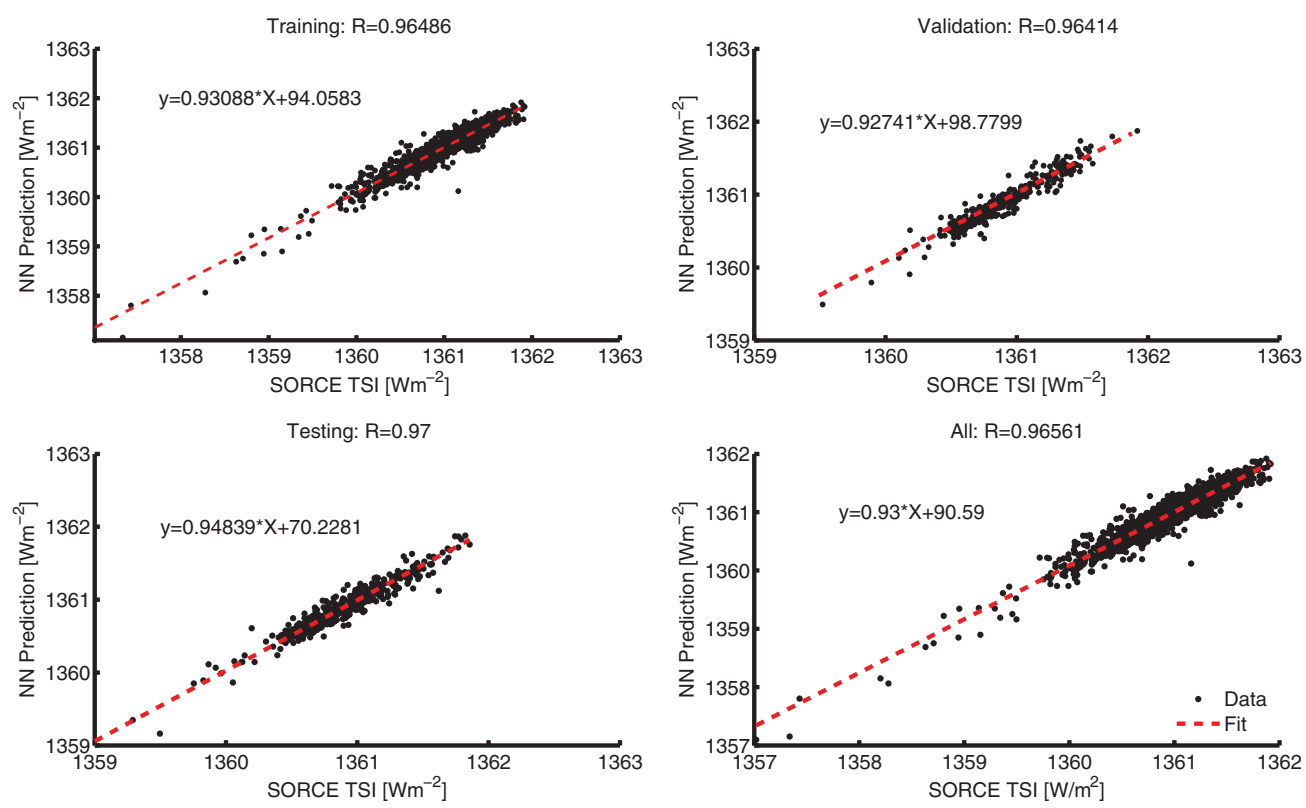

Figure 3. Comparison of the NNs modeling and targets for training, validation and testing data sets.

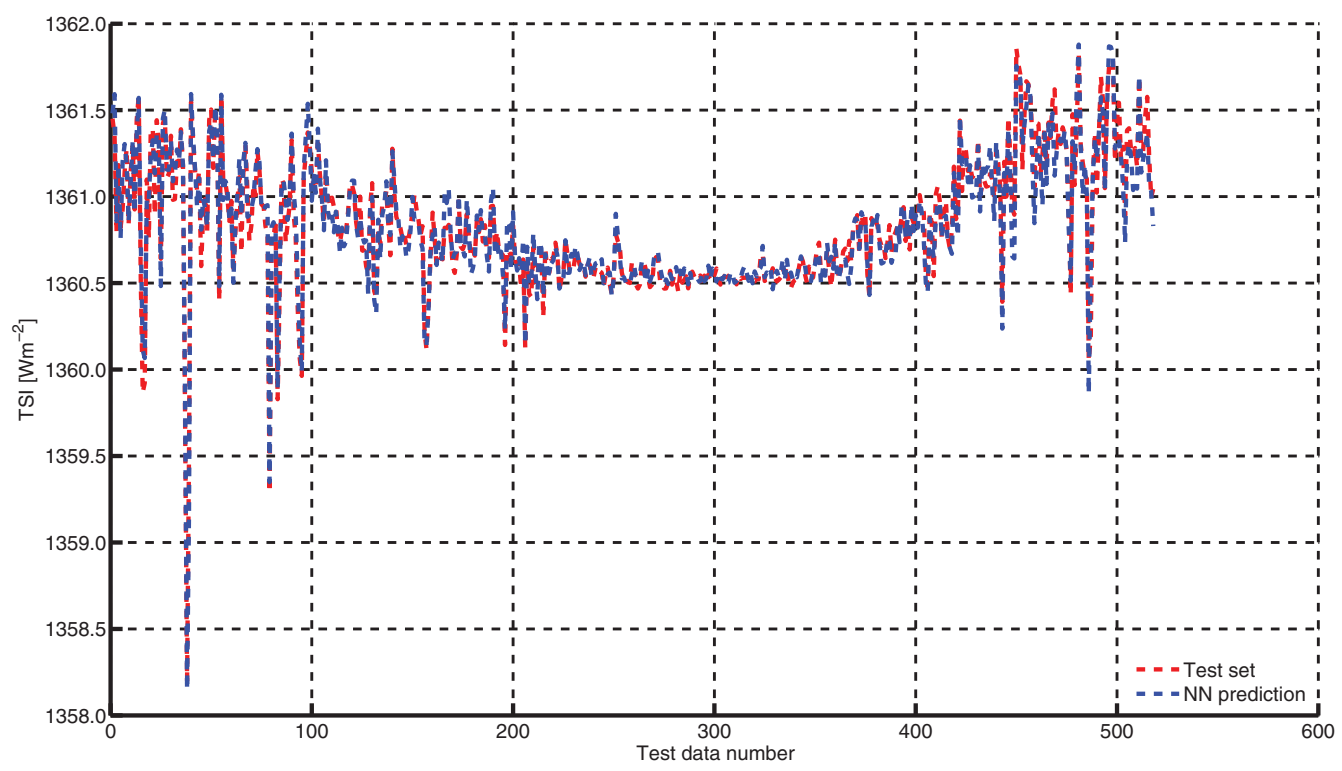

Figure 4. Temporal variations of NNs modeled TSI (blue) and SORCE TSI (red).

SORCE TSI data sets. In the panel, the corresponding correlation coefficient $\mathrm{R}$, and the linear equations are given. This performance is achieved based on 518 data points. The measured TSI and model prediction has a correlation coefficient of $\mathrm{R}=0.97$, and this means that $94 \%$ the total variation of the TSI is due to these solar proxies. 


\section{Conclusions}

In this paper, artificial neural networks method is used to model past TSI from SORCE measurements. The neural network method used here is a static feed-forward network and the Levenberg-Marquardt back-propagation algorithm is adopted for training the $\mathrm{NN}$ to minimize the RMSE and MAE. PSI, $\mathrm{Mg} \mathrm{II} l t$, and $\mathrm{Mg} \mathrm{II}_{s t}$ are chosen as input neuron and TSI from SOURCE as output neuron. The best NN configuration for the present study was found to be a single hidden layer containing 6 neurons. The selection of this configuration was based on the minimization of the difference in RMSE and in MAE between the target and predicted values of TSI. The results can be concluding as follows:

- A comparison between the SORCE TSI and the predicted one using the developed feed-forward network model shows a good performance for the test set data and their correlation coefficient is $\mathrm{R}=0.965$. Therefore, this model has able to explain $94 \%$ of the variation of TSI.

- The dynamic nonlinear trend of SORCE TSI is well reproduced by the model for the year 2003 through 2013.

- The implication of the close correlation between SORCE TSI and the predicted one is that the surface magnetic field causes the irradiance variation on the time scales of days up to solar cycles.

\section{References}

Pap, J., M. \& Fox, P. 2004, Geophysical Monographs Series,11

Lean, J. 2001, Physics Today, 58, 6

Balmaceda, L. A., Solanki, S. K., \& Krivova, N. A. 2009, J. Geophys. Res., 114, A07104

Deland, M. T. \& Cebula, R. P. 1993, J. Geophys. Res., 98

Rottman, G.2006, Space. Sci. Rev., 125, 39

Fröhlich, C. \& Lean, J. 2004, A\&\&AR,4, 12

Balmaceda, L., Krivova, N. A., \& Solanki, S. K. 2007, Adv. Sp. Res.,40,986

Fröhlich, C. \& Lean, J. 1998, Geophys. Res. Lett., 25,4377

Ashamari, O., Qahwaji, R., Ipson, S., Schöll, M., Nibouche, O., \& Haberreiter M.2015, astroph.SR

Hagan, M.T., Demuth, H., \& Beale, M.1995, PWS Publishing Company, Boston, MA

Hornik, K., Stinchcombe, M., \& White, H. 1989, Neural Networks, 2, 359

Habarulema, J. B., McKinnell, L.-A., \& Opperman, B. D. L. 2007, JASTP,72,1842

Habarulema, J. B.,\& McKinnell, L.-A. 2012, Ann. Geophys., 30, 857

Tebabal, A., Baylie, D., Melessew, N., Bires, A., \&Yizengaw, E. 2015, JASTP, 135, 64

Srivastava, N., Hinton, G., Krizhevsky, A., ISutskever, I., \& Salakhutdinov, R. 2014, Journal of Machine Learning Research,15, 1929

Demuth, H. \& Beale, M. 2000, The MathWorks, Inc.

Hoppe, P., Ott, U., \& Lugmair, G. W. 2004, New Astron. Revs, 48, 171

Fröhlich, C. \& Lean, J. 2004, AAR, 4, 273

Kopp, G. \& Lean, J. L. 2011, Geophys. Res. Lett., 28, 1706 